\title{
Backstepping Design for Time-Delay Nonlinear Systems
}

\author{
Frédéric Mazenc, \\ Projet MERE INRIA-INRA, \\ UMR Analyse des Systèmes et Biométrie, \\ INRA 2, pl. Viala, 34060 Montpellier, France, \\ e-mail: mazenc@helios.ensam.inra.fr \\ Pierre-Alexandre Bliman, \\ INRIA Rocquencourt, \\ Projet SOSSO, BP 105, \\ 78153 Le Chesnay cedex, France, \\ e-mail: pierre-alexandre.bliman@inria.fr
}

\begin{abstract}
The backstepping approach is adapted to the problem of globally uniformly asymptotically stabilizing nonlinear systems in feedback form with a delay arbitrarily large in the input. The strategy of design relies on the construction of a Lyapunov-Krasovskii functional. Continuously differentiable control laws are constructed.
\end{abstract}

\section{Introduction}

One of the most popular nonlinear techniques of control design is the backstepping approach. It is presented for instance in $[2,17,3]$ and in $[8$, Chapter 13]. The key ideas of the approach are the following. If for a system in feedback form, i.e. of the form

$$
\left\{\begin{array}{l}
\dot{x}=f(x)+g(x) z \\
\dot{z}=u+h(x, z)
\end{array}\right.
$$

with $x \in \mathbb{R}^{n_{x}}, z \in \mathbb{R}$, where $u \in \mathbb{R}$ is the input and $f(x), g(x), h(x, z)$ are continuous functions, there exists a continuously differentiable function $z_{s}(x)$ such that $z_{s}(0)=0$ and the system

$$
\dot{x}=f(x)+g(x) z_{s}(x)
$$

is globally asymptotically stable and if besides is known a positive definite and radially unbounded function $V(x)$ of class $C^{1}$ such that

$$
W(x):=-\frac{\partial V}{\partial x}(x)\left[f(x)+g(x) z_{s}(x)\right]
$$

is positive definite, then the system (1) is globally asymptotically stabilized by

$$
u(x, z)=-k\left(z-z_{s}(x)\right)-h(x, z)+\frac{\partial z_{s}}{\partial x}(x)[f(x)+g(x) z]-\frac{\partial V}{\partial x}(x) g(x)
$$

A short version of the paper has been accepted by the 42nd IEEE CDC. 
where $k$ is a positive real number. Moreover, the derivative of the Lyapunov function

$$
U(x, z)=V(x)+\frac{1}{2}\left[z-z_{s}(x)\right]^{2}
$$

along the trajectories of (2) in closed-loop with (4) satisfies

$$
\dot{U}(x, z)=-W(x)-k\left[z-z_{s}(x)\right]^{2} .
$$

Many extensions of this basic result have been proved. The multiple advantages offered by this approach are well-known. Observe in particular that this technique yields a wide family of globally asymptotically stabilizing control laws, allows to address robustness issues and to solve adaptive problems.

The objective of the present work is to show how the backstepping approach can be adapted to the problem of stabilizing systems in feedback form with a delay in the input. More precisely, we give sufficient conditions ensuring that a nonlinear system of the form

$$
\left\{\begin{array}{l}
\dot{x}(t)=f(x(t))+g(x(t)) z(t) \\
\dot{z}(t)=u(t-\tau)+h(x(t-\tau), z(t-\tau))
\end{array}\right.
$$

with $x \in \mathbb{R}^{n_{x}}, z \in \mathbb{R}$, where $u \in \mathbb{R}$ is the input and where $\tau$ is a positive real number is globally uniformly asymptotically stabilizable by continuously differentiable state feedback. Roughly speaking, these conditions ensure that the $x$-subsystem with $z$ as virtual input is globally uniformly asymptotically stabilizable by a control law with a delay $\tau$ and that, for a specific family of control laws, the finite escape time phenomenon does not occur. This work completes the families of recent papers devoted to the control of nonlinear systems with delay. In [11] and [13], the technique of [15] is adapted to the problem of stabilizing chains of integrators with bounded controls when there is a delay arbitrarily large in the input. The main result of [11] is extended in [10] to a family of feedforward nonlinear systems. In [12], the problem of stabilizing an oscillator by bounded feedback when there is a delay in the input is solved. In [14], the interconnection of nonlinear systems with delay is studied. In $[6,7]$, the concept of control Lyapunov function is extended to the case of nonlinear systems with delay through the Razumikhin theorem. In [16], connections between Razumikhin-type theorems and the ISS nonlinear small gain theorems are exposed. The present work is distinguished from the papers mentioned above because on the one hand it is devoted to systems in feedback form and on the other hand the key tool we use to prove the main result is the Lyapunov-Krasovskii functional (see [9, 4]). Surprisingly enough, this family of Lyapunov functionals has been used so far mainly in the context of the stabilization of linear systems through linear control laws, see for instance $[1,5]$. We show in this work that it can be also fruitfully exploited to carry out control designs for nonlinear systems. The potential advantages of the knowledge of such a functional are multiple and appealing. Observe in particular that strict Lyapunov functions are known to be very efficient tools for robustness analysis, but this issue is beyond the scope of our work. The stabilizability result we obtain is a global uniform asymptotic stabilizability result for an arbitrarily large delay. The expressions of control laws we exhibit depend on the value of the delay. We want to emphasize that we do not assume that the systems (7) are locally exponentially stabilizable. The example we give in Section 4 to illustrate our control design shows that indeed this assumption is not needed.

Organization of the work. Two technical lemmas are stated and proved in Section 2. In Section 3 the backstepping approach is adapted to the case of systems with delay in the input. An illustrating example is presented in Section 4. Some concluding remarks in Section 5 end the work. 


\section{Definitions and technical preliminaries.}

1. We assume throughout the paper that the functions encountered are sufficiently smooth.

2. The argument of the functions and of the functionals will be omitted or simplified whenever no confusion can arise from the context. For example, one may denote a function $f(x(t))$ by simply $f(x)$ or $f(t)$ or $f(\cdot)$ or $f$.

3. For a real-valued $C^{1}$ function $k(\cdot)$, we denote by $k^{\prime}(\cdot)$ its first derivative.

4. A real-valued function $k(\cdot)$ is of class $\mathcal{K}_{\infty}$ if it is zero at zero, strictly increasing and unbounded.

6. The following norms will be used: $\|\cdot\|$ refers to the Euclidean vector norm; $\|\phi\|_{c}=\sup _{t \in[-r, 0]}\|\phi(t)\|$ stands for the norm of a function $\phi \in C^{1}\left([-r, 0], \mathbb{R}^{m}\right)$.

7. Let $r$ be a positive real number. For a given $t \geq 0, x_{t}(\cdot)$ denotes the restriction of $x(\cdot)$ to the interval $[t-r, t]$ translated to $[-r, 0]$, i.e.

$$
x_{t}(\theta)=x(t+\theta), \quad \forall \theta \in[-r, 0] .
$$

\section{Technical lemmas}

This section is devoted to the construction of Lyapunov-Krasovskii functionals for two families of systems with delay. These constructions are crucial in establishing the main result of the work.

\subsection{First technical lemma}

The following lemma gives conditions ensuring that the $x$-subsystem of (7) with $z$ as virtual input is globally uniformly asymptotically stabilizable by a control law $z_{s}(x(t-\tau))$ and provides with a Lyapunov-Krasovskii functional.

Lemma 2.1 Consider the system

$$
\dot{x}(t)=f(x(t))+g(x(t)) z_{s}(x(t-\tau))
$$

where $x \in \mathbb{R}^{n_{x}}$, where $f(x), g(x)$ are continuous functions, where $z_{s}(x)$ is a continuously differentiable real-valued function such that $z_{s}(0)=0$ and $\tau$ is a positive real number. Assume that there exist a positive definite and radially unbounded function $V(x)$, a positive definite function $W(x)$ and a positive real number $\Omega \geq 8 \tau$ such that the following assumptions are satisfied

H1. For all $x \in \mathbb{R}^{n_{x}}$, the equality

$$
\frac{\partial V}{\partial x}(x)\left[f(x)+g(x) z_{s}(x)\right]=-W(x)
$$

holds.

H2. For all $x \in \mathbb{R}^{n_{x}}$ and $\xi \in C^{1}\left([0,2 \tau], \mathbb{R}^{n_{x}}\right)$, the inequality

$$
-\frac{1}{4} W(x)-T(x, \xi)-\frac{1}{\Omega} \int_{0}^{2 \tau} W(\xi(l)) d l \leq 0
$$

with

$$
\begin{aligned}
T(x, \xi) & =\frac{\partial V}{\partial x}(x) g(x) \int_{\tau}^{2 \tau} H(\xi(l), \xi(l-\tau)) d l \\
H(a, b) & =\frac{\partial z_{s}}{\partial x}(a)\left[f(a)+g(a) z_{s}(b)\right],
\end{aligned}
$$

holds. 
H3. For all function $\xi \in C^{1}\left([0,2 \tau], \mathbb{R}^{n_{x}}\right)$, there exists a contant $K_{\xi} \geq 0$ such that, for all $x \in \mathbb{R}^{n_{x}}$ and for all $t \in[0,2 \tau]$, the inequality

$$
-\frac{1}{2} W(x)+\frac{\partial V}{\partial x}(x) g(x)\left[z_{s}(\xi(t))-z_{s}(x)\right] \leq K_{\xi}[V(x)+1]
$$

holds.

Then the origin of (8) is globally uniformly asymptotically stable. Moreover, along the trajectories of (8), the derivative of the functional

$$
U\left(x_{t}\right)=V(x(t))+\frac{1}{\Omega} \int_{t-2 \tau}^{t}\left(\int_{s}^{t} W(x(l)) d l\right) d s
$$

satisfies, for all $t \geq 2 \tau$,

$$
\dot{U}(t) \leq-\frac{1}{2} W(x(t))
$$

Proof. Let $\varphi \in C^{1}\left([-\tau, 0], \mathbb{R}^{n_{x}}\right)$ be any initial condition of the system (8). Let $T \in(0, \tau]$ be such that the solution $x(t)$ is defined on $[0, T)$. Then, for all $t \in[0, T)$,

$$
\dot{x}(t)=f(x(t))+g(x(t)) z_{s}(\varphi(t))
$$

and

$$
\dot{V}(t)=-W(x(t))+\frac{\partial V}{\partial x}(x(t)) g(x(t))\left[z_{s}(\varphi(t))-z_{s}(x(t))\right] .
$$

From the inequality (12) in H3, we deduce that there exists $K_{\varphi}>0$ such that, for all $t \in[0, T)$

$$
\dot{V}(t) \leq K_{\varphi}[V(x(t))+1] .
$$

It follows that, for all $t \in[0, T)$, the inequality

$$
V(x(t)) \leq(V(x(0))+1) e^{K_{\varphi} t}-1
$$

is satisfied. From this inequality, one can deduce that the finite escape time phenomenon does not occur on $[0, \tau]$; the solutions are well-defined on $[0, \tau]$. By applying this reasoning repeatedly, one can prove that all the solutions are defined on any interval $[k \tau,(k+1) \tau]$, where $k$ is an integer, and thereby on $[0,+\infty[$.

Now, let us observe that, for all $t \geq 2 \tau$, the derivative of the functional $U$ defined in (13) along the trajectories of (8) satisfies

$$
\begin{aligned}
\dot{U}(t) & =\dot{V}(t)-\frac{1}{\Omega}\left(\int_{t-2 \tau}^{t} W(x(l)) d l\right)+\frac{2 \tau}{\Omega} W(x(t)) \\
& =\frac{\partial V}{\partial x}(x(t))\left[f(x(t))+g(x(t)) z_{s}(x(t-\tau))\right]-\frac{1}{\Omega}\left(\int_{t-2 \tau}^{t} W(x(l)) d l\right)+\frac{2 \tau}{\Omega} W(x(t)) .
\end{aligned}
$$

From (9), we deduce that

$$
\begin{aligned}
\dot{U}(t)= & \left(-1+\frac{2 \tau}{\Omega}\right) W(x(t))+\frac{\partial V}{\partial x}(x(t)) g(x(t))\left[z_{s}(x(t-\tau))-z_{s}(x(t))\right]-\frac{1}{\Omega}\left(\int_{t-2 \tau}^{t} W(x(l)) d l\right) \\
= & \left(-1+\frac{2 \tau}{\Omega}\right) W(x(t))-\frac{1}{\Omega}\left(\int_{t-2 \tau}^{t} W(x(l)) d l\right) \\
& -\frac{\partial V}{\partial x}(x(t)) g(x(t)) \int_{t-\tau}^{t} \frac{\partial z_{s}}{\partial x}(x(s))\left[f(x(s))+g(x(s)) z_{s}(x(s-\tau))\right] d s .
\end{aligned}
$$


From the inequality $\Omega \geq 8 \tau$ and (10), we deduce that, for all $t \geq 2 \tau$, the inequality (14) is satisfied. On the other hand, one can check readily that there exist two functions $\mu_{1}, \mu_{2}$ of class $\mathcal{K}_{\infty}$ such that, for all function $\phi \in C^{1}\left([-2 \tau, 0], \mathbb{R}^{n_{x}}\right)$, the functional $U$ satisfies the inequalities

$$
\mu_{1}(\| \phi(0)||) \leq U(\phi) \leq \mu_{2}\left(\|\phi\|_{c}\right)
$$

Finally, from the Krasovskii stability theorem (see [4], [9]), one can deduce that the origin of (8) is globally uniformly asymptotically stable. This concludes the proof.

\subsection{Second technical lemma}

The following lemma establishes the asymptotic stability of a family of linear systems with delay through a Lyapunov-Krasovskii approach.

Lemma 2.2 Consider the system

$$
\dot{Z}(t)=-\varepsilon Z(t-\tau)
$$

where $Z \in \mathbb{R}$ and $\tau$ and $\varepsilon$ are positive real numbers such that $\varepsilon \in\left(0, \frac{1}{2 \tau}\right]$. The origin of this system is globally uniformly asymptotically stable. Moreover, for all $t \geq 2 \tau$, the derivative of the functional

$$
M\left(Z_{t}\right)=\frac{1}{2} Z(t)^{2}+\varepsilon^{3} \tau \int_{t-2 \tau}^{t}\left(\int_{s}^{t} Z(l)^{2} d l\right) d s
$$

along the trajectories of (22) satisfies, for all $t \geq 2 \tau$,

$$
\dot{M}(t) \leq-\frac{1}{4} \varepsilon Z(t)^{2}-\frac{1}{2} \varepsilon^{3} \tau \int_{t-2 \tau}^{t} Z(s)^{2} d s .
$$

Proof. Since the system (22) is linear, its solutions are defined for all $t \geq 0$. One can check readily that the derivative of the quadratic function

$$
L(Z)=\frac{1}{2} Z^{2}
$$

along the trajectories of (22) satisfies, for all $t \geq 2 \tau$,

$$
\begin{aligned}
\dot{L}(t) & =-\varepsilon Z(t) Z(t-\tau) \\
& =-\varepsilon Z(t)^{2}+\varepsilon Z(t)[Z(t)-Z(t-\tau)] \\
& =-\varepsilon Z(t)^{2}-\varepsilon^{2} Z(t) \int_{t-\tau}^{t} Z(s-\tau) d s .
\end{aligned}
$$

Using the triangular inequality, one obtains

$$
\dot{L}(t) \leq-\frac{1}{2} \varepsilon Z(t)^{2}+\frac{1}{2} \varepsilon^{3}\left(\int_{t-\tau}^{t} Z(s-\tau) d s\right)^{2} .
$$

Cauchy-Schwartz inequality leads to

$$
\dot{L}(t) \leq-\frac{1}{2} \varepsilon Z(t)^{2}+\frac{1}{2} \varepsilon^{3} \tau \int_{t-2 \tau}^{t-\tau} Z(s)^{2} d s .
$$


This last inequality implies that the derivative of the functional $M$ defined in (23) along the trajectories of (22) satisfies, for all $t \geq 2 \tau$,

$$
\begin{aligned}
\dot{M}(t) & \leq-\frac{1}{2} \varepsilon Z(t)^{2}+\frac{1}{2} \varepsilon^{3} \tau \int_{t-2 \tau}^{t-\tau} Z(s)^{2} d s-\varepsilon^{3} \tau \int_{t-2 \tau}^{t} Z(s)^{2} d s+\varepsilon^{3} \tau^{2} Z(t)^{2} \\
& \leq \varepsilon\left(-\frac{1}{2}+\varepsilon^{2} \tau^{2}\right) Z(t)^{2}-\frac{1}{2} \varepsilon^{3} \tau \int_{t-2 \tau}^{t} Z(s)^{2} d s
\end{aligned}
$$

From $\varepsilon \in\left(0, \frac{1}{2 \tau}\right]$, one deduces that the inequality (24) holds. On the other hand, one can check readily that, for all function $\phi \in C^{1}([-2 \tau, 0], \mathbb{R})$, the functional $M$ satisfies the inequalities

$$
\frac{1}{2}\|\phi(0)\|^{2} \leq M(\phi) \leq\|\phi\|_{c}^{2}
$$

Finally, from the Krasovskii stability theorem (see [4], [9]), one can deduce that the origin of (22) is globally uniformly asymptotically stable. This concludes the proof.

\section{Main results}

Consider the nonlinear system (7) and introduce a set of assumptions.

Assumption A1. A positive definite, radially unbounded and continuously differentiable function $V(x)$, a continuous and positive definite function $W(x)$, a positive real number $\Omega \geq 8 \tau$, a continuously differentiable function $z_{s}(x)$, satisfying $z_{s}(0)=0$, such that the system

$$
\dot{x}(t)=f(x(t))+g(x(t)) z_{s}(x(t-\tau))
$$

satisfies the assumptions $\mathrm{H} 1$ to H3 of Lemma 2.1 are known.

Assumption A2. Let $C$ be a positive real number. For all $x \in \mathbb{R}^{n_{x}}$, the inequalities

$$
\left|\frac{\partial V}{\partial x}(x) g(x)\right|^{2} \leq W(x),\left|\frac{\partial z_{s}}{\partial x}(x) g(x)\right| \leq C
$$

are satisfied.

Theorem 3.1 Assume that the system (7) satisfies the assumptions A1 and A2. Then the system (7) is globally uniformly asymptotically stabilized by the feedback

$$
u_{s}(t)=-\varepsilon\left(z(t)-z_{s}(x(t-\tau))\right)-h(x(t), z(t))+\frac{\partial z_{s}}{\partial x}(x(t))[f(x(t))+g(x(t)) z(t)]
$$

where $\varepsilon$ is a positive real number such that $\varepsilon \in\left(0, \frac{1}{2 \tau}\right]$.

\section{Discussion of Theorem 3.1.}

- The family of control laws (33) and the family of control laws (4) are different, even when $\tau=0$. The term $-\frac{\partial V}{\partial x}(x) g(x)$ is present in (4) whereas no corresponding term is in (33). The reason is the following. In absence of delay, thanks to the term $-\frac{\partial V}{\partial x}(x) g(x)$, the control law (4) globally asymptotically stabilizes the system (1), even if the functions $V(x), g(x), z_{s}(x)$ do not satisfy growth conditions of the type (32). When a delay is present, control laws with delay of the form (4) globally uniformly asymptotically stabilize the systems (7) only when very restrictive growth properties are satisfied by $V(x), g(x), z_{s}(x)$. Fortunately, under the less restrictive assumptions of Theorem 3.1, the systems (7) are globally uniformly asymptotically stabilized by control laws (33). 
- Assumption A1 is only concerned with the $x$-subsystem of (7). Assumption H1, which ensures the stabilizability of this system with $z$ as virtual input when there is no delay, is not surprising. In the well-known framework of the backstepping for systems without delay, this assumption, or a similar one, is imposed. Assumptions $\mathrm{H} 2$ and $\mathrm{H} 3$ are introduced in order to ensure the stabilizability of the $x$-subsystem of (7) with $z$ as virtual input by a control law with the delay $\tau$.

- In the proof of Theorem 3.1, we shall see that, thanks to Assumption A2, one can construct a Lyapunov-Krasovskii functional for the system (7) in closed-loop with the control law (33) by taking advantage of the two functionals provided by Lemma 2.1 and Lemma 2.2. However, this assumption is not just a convenient tool motivated only by the wish to carry out a Lyapunov construction: It ensures that the finite escape time phenomenon does not occur. It cannot be removed without being replaced by another assumption of a similar type. This fact is enlightened by the system

$$
\left\{\begin{array}{l}
\dot{x}(t)=-x(t)+x(t)^{4} z(t) \\
\dot{z}(t)=u(t-\tau)
\end{array}\right.
$$

because on the one hand, it is globally asymptotically stabilized by the feedback $u(x, z)=-z-x^{5}$ when $\tau=0$, but is not globally uniformly asymptotically stabilizable when $\tau>0$ (see Appendix A) and on the other hand, it is of the form (7), satisfies Assumption A1 with $V(x)=x^{2}, W(x)=$ $2 x^{2}, z_{s}(x)=0$ but, for any choice of functions $V(x)$ and $z_{s}(x)$, it does not satisfy Assumption A2.

- We prove in Appendix B that Assumption A1 may be replaced by the assumption consisting of $\mathrm{H} 1, \mathrm{H} 3$ and the slightly more restrictive assumption:

Assumption H2'. For all $\xi \in C^{1}\left([0,2 \tau], \Re^{n_{x}}\right)$, the inequality

$$
\Omega \tau \int_{\tau}^{2 \tau}|H(\xi(l), \xi(l-\tau))|^{2} d l \leq \int_{0}^{2 \tau} W(\xi(l)) d l
$$

where $H(\cdot)$ is the function defined in (11) and where $\Omega$ is a positive real number such that $\Omega \geq 8 \tau$.

Thanks to Assumptions H2' and A2 one understands that, roughly speaking, a necessary condition for the uniform asymptotic stabilizability of the system $(7)$ is that $z_{s}(x)$ and its first partial derivatives be sufficiently small in norm. In some cases, one can take advantage of Assumption H2' to determine suitable functions $z_{s}(x)$.

- Many extensions of Theorem 3.1 can be easily proved. For instance, the approach applies when the $x$-subsystem of (7) is not affine with respect to $z$, extensions to multi-input systems in feedback form can be made and Theorem 3.1 can be extended to systems of the form

$$
\left\{\begin{array}{l}
\dot{x}(t)=f(x(t))+g(x(t))\left(z(t)+h_{1}(x(t-\tau), z(t-\tau))\right), \\
\dot{z}(t)=u(t-\tau)+h_{2}(x(t-\tau), z(t-\tau))
\end{array}\right.
$$

where $h_{1}(\cdot)$ is a nonlinear function of class $C^{1}$. However, in this section we have chosen to focus our attention on systems of the form (7) because the key ideas of our control design in the case where bounded feedbacks are not searched out can be clearly exposed and understood when applied to (7).

- Theorem 3.1 applies to systems which are not locally linearizable. The assumptions of this theorem do not even ensure that the $x$-subsystem of (7) with $z$ as virtual input is locally exponentially stabilizable. The example we give in Section 4 illustrates this remark.

\section{Proof of Theorem 3.1.}

The proof consists in constructing a Lyapunov-Krasovskii functional whose derivative along the trajectories the system (7) in closed-loop with the control law (33) is smaller than a negative definite function. To simplify the construction, we perform the change of variable

$$
Z(t)=z(t)-z_{s}(x(t-\tau))
$$


which transforms (7) into

$$
\begin{cases}\dot{x}(t) & =f(x(t))+g(x(t))\left(Z(t)+z_{s}(x(t-\tau))\right) \\ \dot{Z}(t) & =u(t-\tau)+\Upsilon(x(t-\tau), x(t-2 \tau), Z(t-\tau)) \\ \Upsilon(a, b, c) & =h\left(a, c+z_{s}(b)\right)-\frac{\partial z_{s}}{\partial x}(a)\left[f(a)+g(a)\left(c+z_{s}(b)\right)\right]\end{cases}
$$

This system in closed-loop with the control law $u=u_{s}(\cdot)$ where $u_{s}(\cdot)$ is the function defined in (33), is

$$
\left\{\begin{array}{l}
\dot{x}(t)=f(x(t))+g(x(t))\left(Z(t)+z_{s}(x(t-\tau))\right), \\
\dot{Z}(t)=-\varepsilon Z(t-\tau) .
\end{array}\right.
$$

We will take advantage of Lemma 2.1 and Lemma 2.2 to prove that the derivative of the LyapunovKrasovskii functional (13) along the trajectories of (39) is smaller than a negative definite function of $(x(t), Z(t))$. Before this analysis, we prove that the finite escape time phenomenon does not occur as follows. (i) The $Z$-subsystem of (39) is linear: Its solutions are defined for all $t \geq 0$. (ii) From Assumption H3 in Lemma 2.1 and $z_{s}(0)=0$, we deduce that there exists $K_{0}>0$ such that, for all $T \in[0, \tau]$, such that the solution is defined on the interval $[0, T)$, the following

$$
\begin{aligned}
\dot{V}(t) & =-W(x(t))+\frac{\partial V}{\partial x}(x(t)) g(x(t))\left[z_{s}(0)-z_{s}(x(t))\right]+\frac{\partial V}{\partial x}(x(t)) g(x(t)) Z(t) \\
& \leq-\frac{1}{2} W(x(t))+K_{0}[V(x(t))+1]+\frac{\partial V}{\partial x}(x(t)) g(x(t)) Z(t)
\end{aligned}
$$

holds. From Assumption A2, we deduce that

$$
\dot{V}(t) \leq K_{0}[V(x(t))+1]+\frac{1}{2} Z(t)^{2} .
$$

Thanks to this inequality, invoking arguments similar to those used in the proof of Lemma 2.1 to establish that the solutions of (8) are defined for all $t \geq 0$, one can prove that the solutions of the $x$-subsystem of (39) are defined for all $t \geq 0$. It follows that the solutions of (39) are defined for all $t \geq 0$.

We study now the sign properties of the derivative of the functional $U$ defined in (13) along the trajectories of (39). Using the key ideas of the proof of Lemma 2.1, one can deduce that, for all $t \geq 2 \tau$,

$$
\dot{U}(t) \leq-\frac{1}{2} W(x(t))+\frac{\partial V}{\partial x}(x(t)) g(x(t)) Z(t)-\frac{\partial V}{\partial x}(x(t)) g(x(t)) \int_{t-\tau}^{t} \frac{\partial z_{s}}{\partial x}(x(s)) g(x(s)) Z(s) d s .
$$

According to Assumption A2, the inequality

$$
\dot{U}(t) \leq-\frac{1}{4} W(x(t))+2\left[Z(t)^{2}+C^{2}\left(\int_{t-\tau}^{t}|Z(s)| d s\right)^{2}\right]
$$

is satisfied. From Cauchy-Schwartz inequality, we deduce that

$$
\dot{U}(t) \leq-\frac{1}{4} W(x(t))+2\left[Z(t)^{2}+\tau C^{2} \int_{t-\tau}^{t} Z(s)^{2} d s\right] .
$$

On the other hand, Lemma 2.2 applies to the $Z$-subsystem of (39) because $\varepsilon \in\left(0, \frac{1}{2 \tau}\right]$. Therefore, for all $t \geq 2 \tau$,

$$
\dot{M}(t) \leq-\frac{1}{4} \varepsilon Z(t)^{2}-\frac{1}{2} \varepsilon^{3} \tau \int_{t-2 \tau}^{t} Z(s)^{2} d s
$$


Combining (44) and (45), one obtains that the derivative of the functional

$$
U_{f}\left(x_{t}, Z_{t}\right)=U\left(x_{t}\right)+K M\left(Z_{t}\right)
$$

where $K$ is a positive real number such that $K \geq \max \left\{\frac{12}{\varepsilon}, \frac{4 C^{2}+1}{\varepsilon^{3} \tau}\right\}$, satisfies the inequality

$$
\dot{U}_{f}(t) \leq-\frac{1}{4} W(x(t))-Z(t)^{2} .
$$

The right-hand side of (47) is smaller than a negative definite function of $(x(t), Z(t))$.

Finally, we prove that there exist two functions $\gamma_{1}$ and $\Gamma_{1}$ of class $\mathcal{K}_{\infty}$ such that, for all function $\left(\phi_{x}, \phi_{Z}\right) \in C^{1}\left([-2 \tau, 0], \mathbb{R}^{n_{x}} \times \mathbb{R}\right)$,

$$
\gamma_{1}\left(\left\|\left(\phi_{x}^{\top}(0), \phi_{Z}(0)\right)^{\top}\right\|\right) \leq U_{f}\left(\phi_{x}, \phi_{Z}\right) \leq \Gamma_{1}\left(\left\|\left(\phi_{x}^{\top}, \phi_{Z}\right)^{\top}\right\|_{c}\right) .
$$

The definitions of the functionals $U_{f}, U, M$ imply that

$$
U_{f}\left(\phi_{x}, \phi_{Z}\right) \geq V\left(\phi_{x}(0)\right)+K L\left(\phi_{Z}(0)\right) .
$$

Since the function $V(x)+K L(Z)$ is positive definite and radially unbounded, according to [8, Lemma 3.5], there exists $\gamma_{1}(\cdot)$ of class $\mathcal{K}_{\infty}$ such that, for all $(x, Z) \in \mathbb{R}^{n_{x}} \times \mathbb{R}$,

$$
V(x)+K L(Z) \geq \gamma_{1}\left(\left\|\left(x^{\top}, Z\right)^{\top}\right\|\right) .
$$

It follows that

$$
U_{f}\left(\phi_{x}, \phi_{Z}\right) \geq \gamma_{1}\left(\left\|\left(\phi_{x}(0)^{\top}, \phi_{Z}(0)\right)^{\top}\right\|\right) .
$$

On the other hand, thanks to (21) and (30), we deduce that

$$
\begin{aligned}
U_{f}\left(\phi_{x}, \phi_{Z}\right) & \leq \mu_{2}\left(\left\|\phi_{x}\right\|_{c}\right)+\left\|\phi_{Z}\right\|_{c}^{2} \\
& \leq \Gamma_{1}\left(\left\|\left(\phi_{x}^{\top}, \phi_{Z}\right)^{\top}\right\|_{c}\right)
\end{aligned}
$$

with

$$
\Gamma_{1}(r):=\mu_{2}(r)+r^{2}, \forall r \geq 0 .
$$

The functions $\gamma_{1}(\cdot)$ and $\Gamma_{1}(\cdot)$ are of class $\mathcal{K}_{\infty}$. Therefore, from the Krasovskii stability theorem, one can deduce that the origin of (7) in closed-loop with the feedback (33) is globally uniformly asymptotically stable. This concludes the proof.

\section{Example}

To illustrate Theorem 3.1, we determine a globally uniformly asymptotically stabilizing feedback for the two-dimensional system

$$
\left\{\begin{array}{l}
\dot{x}(t)=x(t) z(t) \\
\dot{z}(t)=u(t-\tau)
\end{array}\right.
$$

where $\tau$ is an arbitrary positive real number, by applying this theorem. Observe that the linear approximation at the origin of (54) is not asymptotically stabilizable, which implies that the system (54) is not locally exponentially stabilizable by continuous feedback. It follows that linear techniques cannot be of any help for proving the local asymptotic stabilizability of this system.

First, let us check that Assumptions A1 and A2 are satisfied by (54) for functions $V(x)$ and $z_{s}(x)$ suitably chosen. Consider the function

$$
V(x)=\eta \ln \left(1+x^{2}\right)
$$


where $\eta$ is a positive real number. This function is a candidate Lyapunov function: It is a positive definite, radially unbounded function of $x$ and is zero at the origin. Consider the function

$$
z_{s}(x)=-\frac{\omega x^{2}}{1+x^{2}}
$$

where $\omega$ is a positive real number. Then, with the notations of Theorem $3.1, f(x)=0, g(x)=x$ and

$$
\frac{\partial V}{\partial x}(x) g(x)=\frac{2 \eta x^{2}}{1+x^{2}}, \frac{\partial z_{s}}{\partial x}(x)=-\frac{2 \omega x}{\left(1+x^{2}\right)^{2}}, W(x)=\frac{2 \eta \omega x^{4}}{\left(1+x^{2}\right)^{2}} .
$$

Assumption $\mathrm{H} 1$ is satisfied for all $\eta>0, \omega>0$. Consider now, in the particular case we are interested in, the functional

$$
\zeta(x, \xi)=-\frac{1}{4} W(x)-T(x, \xi)-\frac{1}{\Omega} \int_{0}^{2 \tau} W(\xi(l)) d l
$$

with $x \in \Re, \xi \in C^{1}([0,2 \tau], \Re)$ and where $T(x, \xi)$ is the functional defined in the general case in (11). One can check readily that it is nonpositive as follows:

$$
\begin{aligned}
\zeta(x, \xi) & =-\frac{\eta \omega x^{4}}{2\left(1+x^{2}\right)^{2}}-\frac{4 \eta \omega^{2} x^{2}}{1+x^{2}} \int_{\tau}^{2 \tau} \frac{\xi(l)^{2}}{\left(1+\xi(l)^{2}\right)^{2}} \frac{\xi(l-\tau)^{2}}{1+\xi(l-\tau)^{2}} d l-\frac{1}{2} \int_{0}^{2 \tau} \frac{\eta \omega \xi(l)^{4}}{\left(1+\xi(l)^{2}\right)^{2}} d l \\
& \leq \eta \omega \int_{0}^{2 \tau}\left[-\frac{x^{4}}{4 \tau\left(1+x^{2}\right)^{2}}+\frac{4 \omega x^{2}}{1+x^{2}} \frac{\xi(l)^{2}}{\left(1+\xi(l)^{2}\right)^{2}}-\frac{1}{2} \frac{\xi(l)^{4}}{\left(1+\xi(l)^{2}\right)^{2}}\right] d l
\end{aligned}
$$

which implies that

$$
\zeta(x, \xi) \leq 0
$$

when $\omega \in\left(0, \frac{1}{\sqrt{32 \tau}}\right]$. Thus, in this case, Assumption H2 is satisfied. Next, let us observe that, for all $x \in \Re, \xi \in C^{1}([0,2 \tau], \Re)$, the inequality

$$
-W(x)+\frac{\partial V}{\partial x}(x) g(x)\left[z_{s}(\xi(t))-z_{s}(x)\right] \leq 4 \eta \omega
$$

is satisfied. Therefore, Assumption $\mathrm{H} 3$ is satisfied with $K_{\xi}=4 \eta \omega$ for all $\xi$. Thus, we have have shown that Assumption A1 is satisfied. Next, let us prove that Assumption A2 is satisfies, when $2 \eta \leq \omega$. One can prove easily that

$$
\begin{gathered}
\left|\frac{\partial V}{\partial x}(x) g(x)\right|^{2}=\frac{4 \eta^{2} x^{4}}{\left(1+x^{2}\right)^{2}}=\frac{2 \eta}{\omega} W(x) \leq W(x) \\
\left|\frac{\partial z_{s}}{\partial x}(x) g(x)\right| \leq \frac{2 \omega x^{2}}{\left(1+x^{2}\right)^{2}} \leq 2 \omega .
\end{gathered}
$$

We conclude that Assumption A2 is satisfied when $2 \eta \leq \omega$. It follows from the above analysis that Theorem 3.1 applies to the system (54) which implies that the feedback

$$
u_{s}(t)=-\varepsilon\left(z(t)+\frac{\omega x(t-\tau)^{2}}{1+x(t-\tau)^{2}}\right)-\frac{2 \varepsilon x(t)^{2}}{\left(1+x(t)^{2}\right)^{2}} z(t)
$$

when $\omega \in\left(0, \frac{1}{\sqrt{32 \tau}}\right]$ and $0<\varepsilon \leq \frac{1}{2 \tau}$ globally uniformly asymptotically stabilizes the system (54). A possible choice is $\omega=\frac{1}{\sqrt{32 \tau}}, \varepsilon=\frac{1}{2 \tau}$. It yields the following expression of control law

$$
u_{s}(t)=-\frac{1}{2 \tau}\left(z(t)+\frac{1}{\sqrt{32 \tau}} \frac{x(t-\tau)^{2}}{1+x(t-\tau)^{2}}\right)-\frac{1}{\tau} \frac{x(t)^{2}}{\left(1+x(t)^{2}\right)^{2}} z(t) .
$$




\section{Conclusion}

We have carried out the design of globally uniformly asymptotically stabilizing feedbacks for a family of nonlinear systems in feedback form with a delay in the input arbitrarily large. One of the features of the method is that it does not apply only to systems which are locally exponentially stabilizable. The proposed control laws depend explicitly on the value of the delay. We conjecture that the approach can be adapted to the case where an exact knowledge of this value is not available. This issue, as well as robustness and disturbance attenuation issues, discrete-time versions of Theorem 3.1, are some issues that we will pursue in future works.

Acknowledgement. The authors would like to thank Iasson Karafyllis for his helpful suggestions and valuable comments.

\section{References}

[1] P.-A. Bliman, Lyapunov equation for the stability of linear delay systems of retarded and neutral type. IEEE Trans. Automat. Contr., Vol. 47, No 2, 2002, pp. 327-335.

[2] J.M. Coron, L. Praly, Adding an integrator for the stabilization problem. Systems \& Control Letters 17 (1991) 89-104.

[3] R. Freeman, P. Kokotovic, Robust Nonlinear Control Design. Birkhauser, Boston, 1996.

[4] J.K. Hale, S.M. Verduyn Lunel, Introduction to Functional Differential Equations. New York, Springer-Verlag, 1993.

[5] D. Ivanescu, S.I. Niculescu, J.M. Dion, L. Dugard, Control of Distributed Delay Systems with Uncertainties: A Generalized Popov Theory Approach. Kybernetika, Vol. 37, No 3, pp. 325-343.

[6] M. Jankovic, Control Lyapunov-Razumikhin Functions for Time Delay Systems. Proceedings of the 38th CDC. Phoenix, Arizona. December 1999.

[7] M. Jankovic, Control Lyapunov-Razumikhin Functions and Robust Stabilization of Time Delay Systems. IEEE Trans. Automat. Contr., Vol. 46, No 7, 2001, pp. 1048 -1060.

[8] H. Khalil, Nonlinear Systems. 2nd ed. Prentice Hall, 1996.

[9] N.N. Krasovskii, Stability of Motion. Stanford: Stanford University Press, 1963.

[10] F. Mazenc, S. Mondié, R. Francisco, Global Asymptotic Stabilization of Feedforward Systems with Delay in the Input. IEEE Trans. Automat. Contr., Vol. 49, No 5, 2004, pp. 844-850.

[11] F. Mazenc, S. Mondié, S.I. Niculescu, Global Asymptotic Stabilization for Chains of Integrators with a Delay in the Input. IEEE Trans. Automat. Contr., Vol. 48, No 1, 2003, pp. 57 -63.

[12] F. Mazenc, S. Mondié, S.I. Niculescu, Global Stabilization of Oscillators With Bounded Input Delayed. Proceedings of the 42th CDC. Las Vegas. December 2002. To appear in Systems \&3 Control Letters.

[13] W. Michiels, D. Roose, Global Stabilization of Multiple Integrators with Time-Delay and Input Constraints. Proceedings of the 3rd IFAC workshop on Time Delay Systems TDS 2001, Santa Fe, New Mexico, December 2001. 
[14] W. Michiels, R. Sepulchre, D. Roose, Stability of Perturbed Delay Differential Equations and Stabilization of Nonlinear Cascade Systems. SIAM Journal of Control and Optimization 40(3) 2002, pp. 661-680.

[15] A.R. Teel, Semi-global stabilization of minimum phase nonlinear systems in special normal forms. Systems \&3 Control Letters, 19, 1992, pp. 187-192.

[16] A.R. Teel, Connections between Razumikhin-type theorems and the ISS nonlinear small gain theorems. IEEE Trans. Automat. Contr., Vol. 43, No 7, 1998, pp. 960-964.

[17] J. Tsinias, Input to State Stability Properties of Nonlinear Systems and Applications to Bounded Feedback Stabilization Using Saturation. ESAIM: Control, Optimisation and Calculus of Variations. March 1997, Vol. 2, pp. 57-85.

\section{A A delay in the input may cause finite escape time}

We prove here that the system (34) is not globally stabilizable by a continuous feedback $u_{s}(x(t-$ $\tau), z(t-\tau), x(t-2 \tau), z(t-2 \tau))$ when $\tau>0$. To prove this result, we proceed by contradiction. We suppose that, there exist $\tau>0$ and a continuous feedback $u_{s}(x(t-\tau), z(t-\tau), x(t-2 \tau), z(t-2 \tau))$ such that all the solutions of the system (34) in closed-loop with this feedback are defined on $[0,+\infty[$.

Let $\delta$ be a positive real number. Consider as initial condition the function $\varphi=\left(\varphi_{x}, \varphi_{z}\right) \in$ $C^{1}\left([-2 \tau, 0], \Re^{2}\right)$ such that $\left(\varphi_{x}(t), \varphi_{z}(t)\right)=(0,0)$ for all $t \leq-\frac{2 \tau}{3},\left(\varphi_{x}(t), \varphi_{z}(t)\right)=(\delta, \delta)$ for all $t \in\left[-\frac{\tau}{3}, 0\right]$ and $\varphi_{x}$ and $\varphi_{z}$ are nondecreasing. Then, for all $t \leq \frac{4}{3} \tau$, the solution of (34) satisfies

$$
\left\{\begin{array}{l}
\dot{x}(t)=-x(t)+x(t)^{4} z(t) \\
\dot{z}(t)=u_{s}(0,0,0,0)
\end{array}\right.
$$

This implies that, for all $t \in\left[0, \frac{4}{3} \tau\right]$,

$$
\left\{\begin{array}{l}
\dot{x}(t)=-x(t)+x(t)^{4} z(t) \\
z(t)=u_{s}(0,0,0,0) t+z(0)=u_{s}(0,0,0,0) t+\delta .
\end{array}\right.
$$

Choosing $\delta$ such that $-\frac{4}{3} \tau\left|u_{s}(0,0,0,0)\right|+\delta \geq \frac{1}{2} \delta$, we have

$$
\dot{x}(t) \geq-x(t)+\frac{1}{2} \delta x(t)^{4}
$$

for all $t \in\left[0, \frac{4}{3} \tau\right]$. It follows that $X(t)=e^{t} x(t)$ satisfies, for all $t \in\left[0, \frac{4}{3} \tau\right]$,

$$
\dot{X}(t) \geq \frac{1}{2} \delta e^{-3 t} X(t)^{4} \geq \frac{1}{2} \delta e^{-4 \tau} X(t)^{4} .
$$

By integrating this inequality, we deduce that, for all $t \in\left[0, \frac{4}{3} \tau\right]$,

$$
X(t)^{3} \geq \frac{1}{\frac{1}{X(0)^{3}}-\frac{\delta}{2} e^{-4 \tau} t}=\frac{\delta^{3}}{1-\frac{\delta^{4}}{2} e^{-4 \tau} t} .
$$

When $\delta \geq\left(\frac{3}{2 \tau}\right)^{\frac{1}{4}} e^{\tau}$, then $\frac{2}{\delta^{4}} e^{4 \tau} \in\left[0, \frac{4}{3} \tau\right]$. It follows that

$$
\sup _{\left\{s \in \left[0, \frac{2}{\delta^{4}} e^{4 \tau}[\}\right.\right.} X(s)^{3} \geq \sup _{\left\{s \in \left[0, \frac{2}{\delta^{4}} e^{4 \tau}[\}\right.\right.} \frac{\delta^{3}}{1-\frac{\delta^{4}}{2} e^{-4 \tau} s}=+\infty .
$$

According to the initial assumption, $x(t)$ and therefore $X(t)$ are continuous functions over $[0,+\infty[$. This contradicts the initial assumption. The finite escape time phenomenon occurs. This concludes the proof. 


\section{B Assumption H2'}

We show that if a system (7) satisfies Assumptions H1, H2', H3 and A2, then it satisfies Assumption $\mathrm{H} 2$ as well. Consider the functional

$$
\zeta(x, \xi)=-\frac{1}{4} W(x)-\frac{\partial V}{\partial x}(x) g(x) \int_{\tau}^{2 \tau} H(\xi(l), \xi(l-\tau)) d l-\frac{1}{\Omega} \int_{0}^{2 \tau} W(\xi(l)) d l .
$$

Using the triangular inequality, one can prove that the inequality

$$
\zeta(x, \xi) \leq-\frac{1}{4} W(x)+\frac{1}{4}\left|\frac{\partial V}{\partial x}(x) g(x)\right|^{2}+\left|\int_{\tau}^{2 \tau} H(\xi(l), \xi(l-\tau)) d l\right|^{2}-\frac{1}{\Omega} \int_{0}^{2 \tau} W(\xi(l)) d l
$$

holds. From Assumption A2, it follows that

$$
\zeta(x, \xi) \leq\left|\int_{\tau}^{2 \tau} H(\xi(l), \xi(l-\tau)) d l\right|^{2}-\frac{1}{\Omega} \int_{0}^{2 \tau} W(\xi(l)) d l .
$$

Cauchy-Schwartz inequality implies that

$$
\zeta(x, \xi) \leq \tau \int_{\tau}^{2 \tau}|H(\xi(l), \xi(l-\tau))|^{2} d l-\frac{1}{\Omega} \int_{0}^{2 \tau} W(\xi(l)) d l .
$$

Assumption H2' implies that

$$
\zeta(x, \xi) \leq 0
$$

Therefore Assumption H2 is satisfied. This concludes the proof. 